\title{
Early Microfilament Reorganization in Injured Auditory Epithelia
}

\author{
Yehoash Raphael and RichaRd A. AltschuleR \\ Kresge Hearing Research Institute, University of Michigan Medical School, Ann Arbor, Michigan 48109
}

\begin{abstract}
Microfilaments (MFs) play an important role in wound healing and other regenerative events. The purpose of this study was to characterize changes in the distribution of MFs in traumatized auditory epithelia and compare these changes between avian (regenerating) and mammalian (nonregenerating) ears. Chicks and guinea pigs were acoustically overstimulated and their auditory epithelia analyzed using fluorescence microscopy with phalloidin as a MF-specific marker. Immediately or several hours after overstimulation, we observed a substantial reduction of MFs in stereocilia and the cuticular plate. The circumferential belt of MF which is associated with the adherens junctional complex was constricted in damaged hair cells (HCs) as early as 1 day after the exposure. Concomitant with the junctional constriction, the apical surface area of supporting cells was increased relative to normal, whereas the surface area of HCs was decreased. We conclude that changes in the amount and distribution of MFs which characterize early responses to acoustic damage are similar in avian (regenerating) and mammalian (nonregenerating) auditory epithelia. We hypothesize that changes in MF-mediated tensile forces trigger the process of tissue repair in auditory epithelia in response to insult. In mammals the reorganization of MFs may help maintain the integrity of the reticular lamina and thereby prevent further damage. In contrast, early changes in MFs in chicks may play a role in regulating regenerative tissue responses. () 1992 Academic Press, Inc.
\end{abstract}

\section{INTRODUCTION}

The molecular mechanisms which govern the response of auditory epithelia to acoustic overstimulation are unknown. Nevertheless, tissue damage resulting from acoustic trauma in avian and mammalian inner ear is well characterized. In mammals, acoustic trauma leads to the death of hair cells $(\mathrm{HCs})$ in the organ of Corti, after which the auditory epithelium replaces lost HCs with scars made by supporting cells (SCs) $(1,5,8)$. This scarring process is irreversible and results in a permanent threshold shift. In contrast, when chickens and other nonmammalian vertebrates are experimentally deafened by exposure to ototoxic drugs or noise over- stimulation, end organ structure and hearing may be restored $(2,9,12)$. 'This repair mechanism involves production of new HCs which replace lost cells.

It is currently unknown whether the early course of acoustic injury differs between avian and mammalian auditory epithelium. It is possible that the effects of overstimulation in regenerating and nonregenerating auditory epithelia share common early mechanisms which may result in different secondary tissue responses. The aim of this study was to determine early changes which occur in avian and mammalian auditory epithelia after acoustic trauma at the structural and molecular levels. We assayed changes in the organization of actin microfilaments (MF) in the apical region of $\mathrm{HCs}$ and SCs following acoustic overstimulation. We chose to study the MF system for the following reasons:

A. MFs are abundant in three domains of the apical region of HCs: stereocilia, cuticular plate (terminal web), and the adherens-type junctional belt (11). Therefore, the distribution of MF label can be used to study the presence and condition of stereocilia and the boundaries of cells in the reticular lamina.

B. Microfilaments have been shown to be important in tissue repair and wound healing in a variety of tissues $(4,6)$.

We found that overstimulation of both chicken and guinea pig auditory epithelia results in depolymerization of MFs in the stereocilia and cuticular plate and constriction of the circumferential MF belt in HCs. Overstimulation also results in expansion of SCs. These findings are qualitatively similar in chicks and guinea pigs, suggesting that early stages of acoustic trauma share several common features among avian (regenerating) and mammalian (nonregenerating) auditory epithelia.

\section{MATERIALS AND METHODS}

Twenty chick hatchlings were acoustically overstimulated with a pure tone of $1.5 \mathrm{kHz}$ at $124 \mathrm{~dB}$ SPL for 10 h. They were killed either immediately after acoustic exposure or $6 \mathrm{~h}$ later. Eight pigmented guinea pigs were exposed to a $4-\mathrm{kHz}$ octave band noise at $117 \mathrm{~dB}$ SPL for 


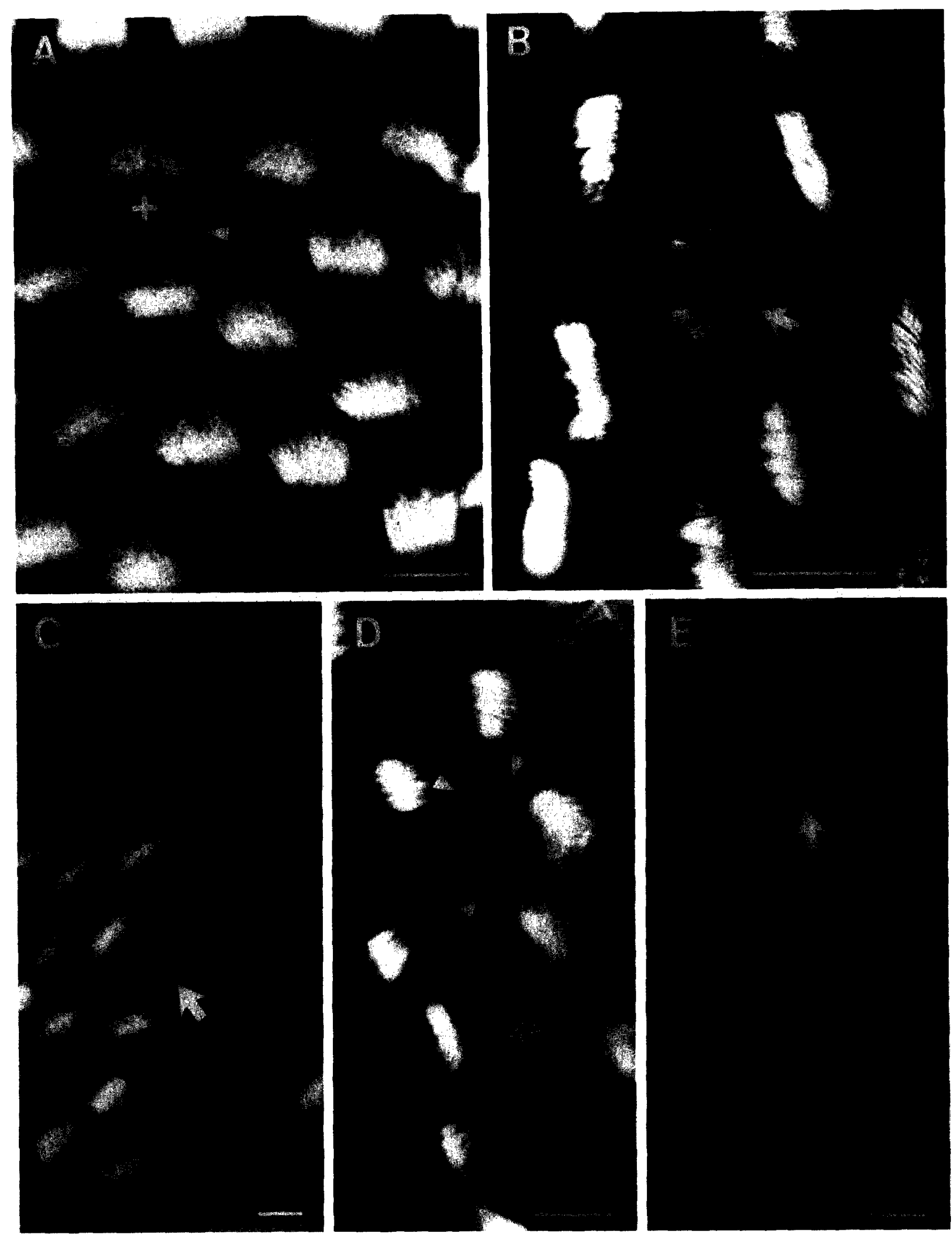

FIG. 1. Surface view of phalloidin-labeled whole-mounts of control (A) and acoustically overstimulated avian auditory epithelia (B-E). (A) MF-specific staining is found in stereocilia (s), cuticular plate $(+)$, and circumferential adherens junction belt (arrowhead). (B) In damaged cells (arrow) several stereocilia are missing in center of bundle while some other stereocilia appear elongated. (C) Several hair cells no longer have stereocilia (arrow). SCs are expanded. (D) Expanded SCs (arrowheads) surround HCs without apparent MF loss (compare to normal $\mathrm{SCs}$, arrow-frame). (E) Six hours after overstimulation, circumferential MF belt of HCs are in final stage of constriction (arrow). Surrounding SCs are expanded and continuity of reticular lamina is maintained. Bars, $10 \mu \mathrm{m}$. 


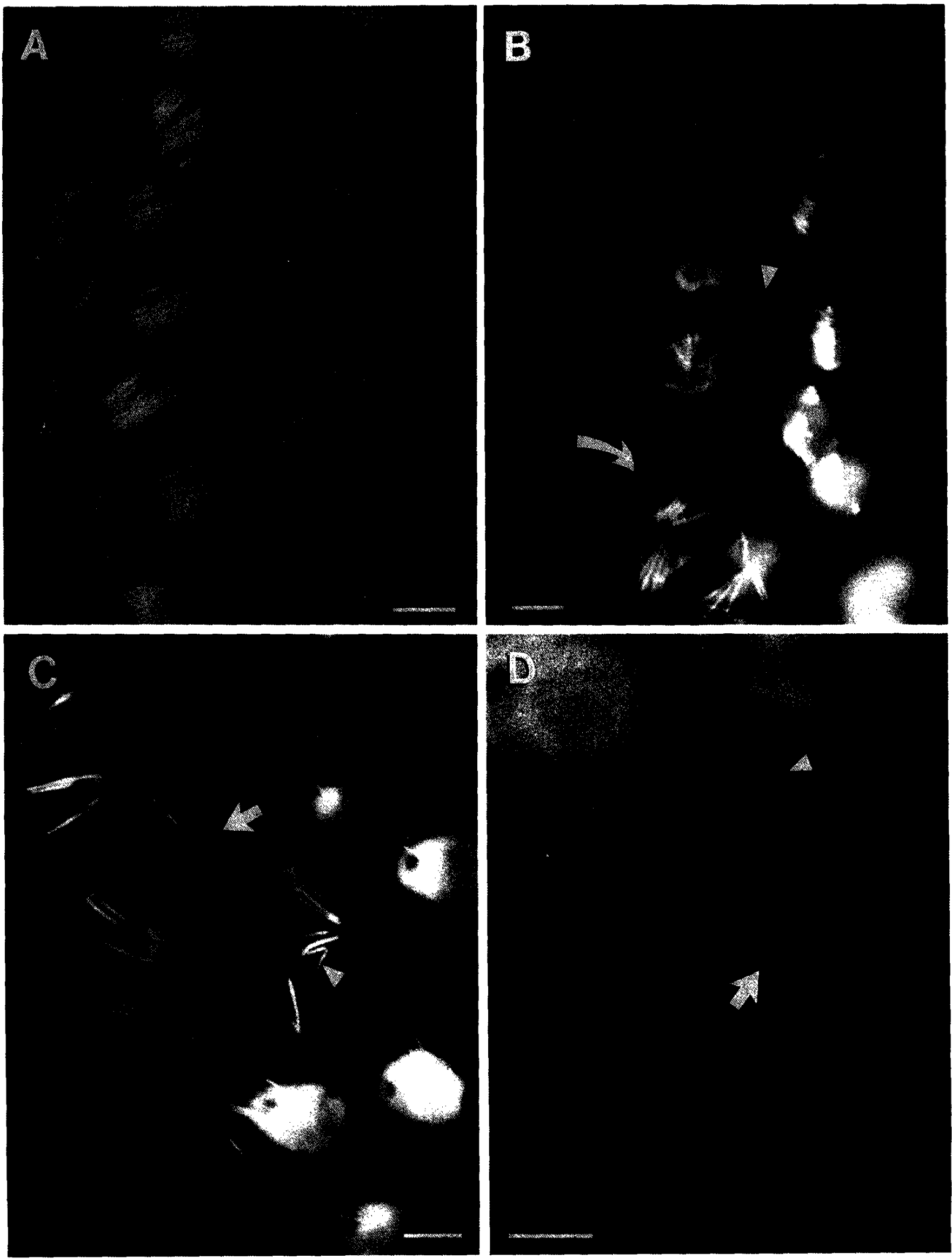


$30 \mathrm{~min}$. Four guinea pigs were killed immediately after exposure and the rest 1 day later.

Dissection of the tissues was carried out essentially as previously described $(7,8)$. To label microfilaments for fluorescence microscopy, whole-mounts of chick basilar papillae or half-turns of guinea pig organ of Corti were fixed with $3 \%$ paraformaldehyde, permeabilized in Triton $\mathrm{X}-100$, and incubated for $30 \mathrm{~min}$ in rhodaminephalloidin (1:50 in PBS). Specimens were mounted in a glycerol-PBS media and photographed in a Leitz Orthoplan microscope equipped for epifuorescence.

\section{RESULTS}

Chick. In a surface view of a normal (unexposed) avian auditory mosaic, phalloidin labeled the stereocilia, cuticular plate, and intercellular junctions between HCs and SCs (Fig. 1A). We observed various degrees of stereocilia loss after $10 \mathrm{~h}$ of pure tone exposure (Figs. 1B and $1 \mathrm{C}$ ). A few of the remaining stereocilia appeared elongated and the apical surface of many SCs had expanded (Fig. 1D). Expanded SCs surrounded HCs which had reduced apical surface area. Six hours after exposure, the apical surface of $\mathrm{HCs}$ in the damaged region was almost completely invaded by neighboring $\mathrm{SCs}$ (Fig. 1E). Missing HCs were replaced by the expanded SCs. The integrity and continuity of the epithelial surface (reticular lamina) were maintained throughout the process of HC replacement.

Guinea pig. MFs in the normal guinea pig auditory mosaic are distributed in stereocilia, cuticular plate, and adherens-type junctions (Fig. 2A). We observed varying degrees of MF loss in stereocilia following acoustic overstimulation. Immediately after acoustic overstimulation, some or all the stereocilia were missing on several HCs. Some stereocilia were elongated to more than twice their original length (Fig. 2B), whereas others were kinked or bent. In addition, loss of label in the cuticular plate was frequently observed (Fig. 2C). One day after acoustic exposure some $\mathrm{HCs}$ exhibited neither stereocilia nor a cuticular plate, but contained a loop of MFs parallel to the reticular lamina at the level of the intercellular junctions (Fig. 2D). Transmission electron microscopy enabled us to identify this MF loop as the constricted circumferential MF belt of the HC (results not shown).

\section{DISCUSSION}

The reorganization of both MFs and intercellular junctions during scar formation in the organ of Corti have been previously characterized (8). The present work demonstrates early changes in the MF cytoskeleton of auditory epithelia observed immediately after acoustic overstimulation. We characterized the reorganization of MFs during injury to the avian auditory epithelium and found several similarities with the mammalian inner ear.

We observed constriction of the apical surface of avian $\mathrm{HCs}$, an event which probably resulted from constriction of the circumferential MF belt associated with the adherens junctions. This constriction coincided with expansion of surrounding $\mathrm{SCs}$, as previously described (3). We did not observe holes in the avian reticular lamina during constriction of $\mathrm{HCs}$ and expansion of SCs. It appears, therefore, that changes in the apical region of $\mathrm{HCs}$ and supporting cells after acoustic trauma are highly coordinated to prevent disruption in the reticular lamina. In mammals, a mechanism for replacing HCs with SCs without disruption of the reticular lamina has been described (8) but in some studies, hole formation has been reported (1).

Damage to MFs in stereocilia after acoustic overstimulation has been observed using transmission electron microscopy (10). Using the fluorescent probe phalloidin, we were able to analyze the distribution of MFs in each stereocilium on the entire stereociliary bundle and thereby observe several patterns of MF depolymerization in stereocilia early after acoustic trauma. We found that some stereocilia were bent or kinked following acoustic overstimulation. This result suggests that crosslinks, which in normal stereocilia bind individual MFs together, are disrupted after acoustic overstimulation and that destruction of MF crosslinks in these stereocilia may precede MF depolymerization. We also identified elongated stereocilia after overstimulation, suggesting that MF-capping molecules, which in normal stereocilia prevent elongation, may have been damaged.

Because some expanding SCs "invade" the space of $\mathrm{HCs}$ which retain normal MF distribution, it appears that $\mathrm{SCs}$ respond to a signal which is undetected in this study. The possibility that overstimulation can trigger a direct effect on SCs cannot be ruled out.

FIG. 2. Surface view of a phalloidin-labeled whole-mount of control (A) or noise-exposed (B-D) guinea pig organ of Corti. (A) In second and third row outer $\mathrm{HCs}$ and SCs around them, MF label is in stereocilia, cuticular plate, and adherens junctions. Note MF-free zone in cuticular plate (basal body area, arrow). (B) Immediately after noise exposure several stereocilia are missing and MF label is reduced in cuticular plate (arrow). Elongated stereocilia are often found (arrowhead). (C) Stiffness is reduced in several stereocilia and many are bent (arrowhead). Stereocilia on some cells are in various stages of degeneration (arrow). (D) One day after insult, several cells have neither stereocilia nor cuticular plate. A loop of MFs is in a centrally located position (arrowhead). Cells which retain a circumferential MF belt (arrow) do not have a MF loop. Bars, $5 \mu \mathrm{m}$. 
We speculate that tensile forces in the reticular lamina are altered during injury to the MFs and that changes in these forces trigger cell-mediated repair. Alteration of tensile forces by MF have been described during trauma in other types of epithelia (6). It is possible that constriction of the apical surface of HCs during trauma occurs in response to altered tensile forces in the reticular lamina.

These data suggest that reorganization of MFs in auditory epithelia occurs very early during acoustic trauma via a mechanism which is similar in avian and mammalian ears. This observation contrasts later tissue response to acoustic trauma, which differs between mammals and birds. Avian species replace lost $\mathrm{HCs}$, whereas mammals do not and instead form scars. Future studies will help elucidate the stage at which response to acoustic trauma in avian and mammalian ears diverge.

\section{ACKNOWLEDGMENTS}

Supported by NIH Grant NS 05785 and the Deafness Research Foundation.

\section{REFERENCES}

1. BOHNE, B. A. 1976. Healing of the noise damaged inner ear. Hearing and Davis: Essays Honoring Hallowel Davis. (S. K. Hirsh, D. H. Eldredge, I. J. Hirsh, and S. R. Silverman, Eds.), pp. 85-96. Washington Univ. Press, Saint Louis.

2. CoRWIN, J. T., AND D. A. COTANCHE. 1988. Regeneration of sensory hair cells after acoustic trauma. Science 240: 1772-1774.
3. Cotanche, D. A., And C. E. J. Dopyera. 1990. Hair cell and supporting cell response to acoustic trauma in the chick cochlea. Hear. Res. 46: 29-40.

4. Gabbiani, G., F. H. Gabbiani, and S. M. Schwartz. 1984. Organization of actin cytoskeleton during endothelial regeneration in vitro. J. Cell Sci. 66: 39-50.

5. Hawkins, J. E., JR., AND L-G. JoHNSSON. 1981. Histopathology of Cochlear and Vestibular Ototoxicity in Laboratory Animals. In Aminoglycoside Ototoxicity (S. A. Lerner, J. G. Matz, and J. E. Hawkins Jr., Eds.), pp. 175-195. Little, Brown, Boston.

6. HergotT, G. J., M. SANDiG, AND V. I. KalNins. 1989. Cytoskeletal organization of migrating retinal pigment epithelial cells during wound healing in organ culture. Cell Motil. Cytoskeleton 13: 83-93.

7. RaPhael, Y. 1991. Damage to the tectorial membrane may protect chick hair cells from noise overstimulation. Hear. Res. 53: 173-184.

8. RAPhafl, Y., AND R. A. Altschuler. 1991. Modulation of cytoskeletal and junctional proteins during hair cell degeneration. Cell Motil. Cytoskeleton 18: 215227.

9. RYALS, B. M., AND E. W. RUBEL. 1988. Hair cell regeneration after acoustic trauma in adult Coturnix quail. Science 240: 1774-1776.

10. Tilney, L. G., J. C. Saunders, E. Egelman, and D. J. DeroSIER. 1982. Changes in the organization of actin filaments in the stereocilia of noise-damaged lizard cochleae. Hear. Res. 7: 181197.

11. Tilney, L. G., AND J. C. SAunders. 1982. Actin filaments, stereocilia, and hair cells of the bird cochlea. I. The length, number, width, and distribution of stereocilia of each hair cell are related to the position of the hair cell on the cochlea. J. Cell Biol. 96: $807-821$

12. TUCCI, D. L., AND E. W. RuBEL. 1990. Physiologic status of regenerated hair cells in the avian inner ear following aminoglycoside ototoxicity. Otolaryngel. Head Neck Surg. 103: 443-450. 